\title{
Research on ESD Protection Design for Electronic Communication Products
}

\author{
Yuan Ke \\ Nanchang Institute of Science \& Technology
}

Keywords: Electronic; Communication Products; ESD Protection Design Technology; Research; Reduce Harm

\begin{abstract}
With the development of society and the progress of the times, people's living standards continue to improve and the electronic communication products also unceasing renew accordingly. Electronic communication products plays a extremely important role in people's daily life. Due to ESD has certain harm to electronic communication products, when using electronic communications products, we shall prevent ESD electrostatic accumulation. To mainly solve the problems caused by ESD, this paper deeply studies ESD protection design of electronic communication products to ensure that the harms of ESD can be reduced to minimum and to ensure the personal and property safety for the people who are using electronic communication products.
\end{abstract}

\section{Introduction}

The meaning of ESD is electro-static discharge. ESD protection is the preventive measures taking to prevent the explosion, fire, electric shock, as well as damage and failure of electronic device and the adverse effects to production caused by the accumulation of static electricity. The precautionary principle is mainly to suppress the production of electrostatic, accelerate electrostatic leakage and carry out electrostatic neutralization. Because walking and other activities will produce and accumulate charge which can reach potential kilovoltage, if people wear non conductive shoes. Two different objects contact with each other, mobile charge produce in the interface, positive static electricity charge protects negative charge which form into relative arrangement and electric double layer. If the objects are separated, equivalent charge of different polarity will product on the two objects.

Due to the rapid development of technology, the integrated degree of electronic devices become more and more high, the volume of electronic products is getting smaller and smaller, and the relatively smaller electronic components are very sensitive to the larger ones. The changes near magnetic field caused by electrostatic discharge will be result in that the working electronic devices get the wrong signal or do the wrong action, which leads to great damage to electronic devices. The protection of ESD is very important. This paper describes the harm brought by ESD in electronic communication products and the preventive measures taken to prevent the adverse effects caused by the accumulation of static electricity. This paper analyzes the harm brought to electronic communication products by ESD, the shortages of the existed ESD protection design scheme, the problems which shall be paid attention to in ESD protection design of electronic communications products and the basic requirements of ESD protection of electronic communications products.

\section{The Harm of ESD in Electronic Communication Products}

The harm of ESD in the electronic communication products is very large, probably including the follows:

Transmit Error Message. ESD has large effects on electronic communications products and the false information indicating appears. For example: if pulse interference in ESD discharges in electronic communication products, the legacy pulse will be detected, which will interfere the information transmission of electronic communication products, which will cause errors, repeat, delay phenomena of electronic products. In this project, pulse will couple with the circuit design of electronic communication products, which will increase the burden of information transmission. 
Since the accumulation of error information in electronic communication products will impede the original smooth of the internal path. So the product is facing hardware impact damage. Thus, the ham of ESD to electronic communication products shall be avoided effectively to ensure the high quality application of electronic communication products.

Component Failure. Component failure belongs to the direct damage, especially in the process of electrostatic discharge. It affects a wide range of electronic communication products around the magnetic field. In magnetic fluctuations, the device will gradually fail, resulting in electronic communication products send or receive abnormal signals and cannot maintain the normal operation. For example: the components of an electronic communication product under the influence of ESD have failure risks. Once the product is operated, the phenomena of crash and collapse will appear.

Component Pollution. ESD has significant adsorption in electronic communication products, which can absorb the floating matters in the air and promote them be concentrated on the surface of electronic communication products, which affects the insulating ability and reduces the working efficiency of electronic communication products. The ESD element pollution exists barrier problems which will cause product failure to a large extent.

\section{Disadvantages of Existing ESD Protection Design Schemes}

The existed ESD protection case has the problems of the distinction between ESD and EOS (Electrical Overstress) and the identification of system types.

EOS is the short form of Electrical Over- Stress with the meaning of voltage failure which is the destruction of electronic components of the thermal effect generated by voltage. In general, from the perspective of the electrostatic bad, the most commonly seen are ESD and EOS, in which ESD meaning refers to the situation of the damage caused by the external static magnetic and the short time partial discharge of ESDS Item itself; while EOS refers to the situation of the damage caused by the improper design of voltage and current or the leakage current of other test machine, production machines, equipment, fixtures and other appliances.

In the formation of the reasons, ESD and EOS are very different, so the analysis comparison basis are also different. Under the conditions of the majority, the current generated by EOS is far greater than the current generated by ESD. In terms of the failure time, ESD and EOS also have very big difference. The failure time of EOS is far greater than that of ESD. As a result, seeing from the phase analysis to the electronic components which are damaged by them, the failure degree of EOS is far more serious than that of ESD.

\section{ESD Protection Design in Electronic Communication Products}

The protection design of ESD shall take the harm of ESD to electronic communication products as the starting point and shall be carefully conducted through the deep analysis its harmfulness.

ESD Protection Design of Casing in Electronic Communication Products. In the part of the electronic communication products, chassis plays an important role and the protection ability of ESD of chassis has a direct impact on the protective ability of the electronic communication products. Only the chassis of electronic communication products reach a certain protective ability, will increase the speed of ESD electrostatic discharge, which makes that there will be no accumulation of a large number of electrostatic charge in electronic communication products, so as to effectively improve the anti-interference ability of electronic communication products. Attention shall be paid to the chassis shall be far away from the internal work environment of electronic communication products and the grounding line, which can effectively prevent ESD harm of electronic communication products and can ensure the self protection of the chassis.

ESD Protection Design for Metal Parts in Electronic Communication Products. In the whole electronic communication products, metal parts occupies a large proportion, which is the core of electronic communication products and is mainly responsible for the communication of the basic business. In design of the internal metal parts of electronic communication products, the metal parts 
shall be isolated to a certain degree to ensure insulation of metal parts. The outside of the metal parts shall be coated with insulating layer to strengthen the ESD protection level of metal parts, so as to improve the protection ability of electronic communication products.

ESD Protection Design of Circuit Board in Electronic Communication Products. In the circuit board of electronic communication products, there are a lot of loops with the fixed performance. These loops are sensitive, when the pulse of electronic communication products meet the loops, great disturbances will produce to the electronic communication products, which makes the electronic communication products transfer wrong information. Therefore, we should improve the protection ability of circuit board ESD. The circuit board shall be as narrow as possible, which can exclude the influence of ESD. Removal of the redundant and useless lines in the circuit board can guarantee the communication quality of the circuit board and prevent interference of of electrostatic charge to the operation of electronic communication products.

\section{Conclusion}

In the working process of the electronic communication products, ESD can produce a certain harm, which will not only influence the working efficiency of electronic communication products, but also will affect the production efficiency of the production enterprises of electronic communication products and the social influence of electronic communication products production enterprises, therefore, the ESD protection design in the production process of electronic products is a very necessary link. In short, ESD protection design work shall take its harmfulness as the starting point and the target. ESD protection standards shall be developed according to the actual needs of the different electronic communication products. ESD protection design shall be carried out deeply and try to improve the protection ability of ESD of electronic communication products as far as possible.

\section{References}

[1] Rapp R. Robust modular electronic device without direct electrical connections for inter-module communication or control: US, US7322863 [P]. 2008.

[2] Ninh L, Lester G. Inflight entertainment system having EMI and ESD improvements: US, US5705860 [P]. 1998.

[3] Meleis H. Network models, methods, and computer program products for managing a service independent of the underlying network technology: WO, US8676944 [P]. 2014.

[4] Leone M, Caprella E E. Method and system for the cipher key controlled exploitation of data resources, related network and computer program products: WO, US 20070079142 A1 [P]. 2007.

[5] Leone M, Caprella E E. Method and system for the cipher key controlled exploitation of data resources, related network and computer program products: WO, US 20070079142 A1 [P]. 2007.

[6] Su Q, Ni M, Tang Z, et al. ESD/antenna diodes for through-silicon vias: US, US8264065 [P]. 2012.

[7] Mejyr S. ESD-detector: EP, US7821751 [P]. 2010.

[8] Chen J, Contreras S. LAN magnetic interface circuit with passive ESD protection: US, US 20040257743 A1 [P]. 2004.

[9] Rapp R J. Extensible robust modular electronic device without direct electrical connections for inter-module communication or control: US, US7727035 [P]. 2010.

[10]Arndt B, Zur Nieden F, Mueller F, et al. Virtual ESD testing of automotive electronic systems[C]// Electromagnetic Compatibility (APEMC), 2010 Asia-Pacific Symposium on. 
IEEE, 2010:683-686.

[11]Bandy Iv W T, Boday D J, Iben I E T, et al. Low viscosity electrostatic discharge (ESD) dissipating adhesive: US, US8493699[P]. 2013.

[12]Imtiaz S S M. Method of accurate prediction of electrostatic discharge (ESD) performance in multi-voltage environment: US, US7987085 [P]. 2011. 